\title{
The Incomplete Exact Inverse Problem of the Calculus of Variations
}

\author{
Veronika Chrastinová \\ Brno University of Technology, Faculty of Civil Engineering, Department of Mathematics, \\ Veveří 331/95, 60200 Brno, Czech Republic \\ Email: chrastinova.v@fce.vutbr.cz
}

\begin{abstract}
In the common theory of the inverse problem, a system of differential equations is given and we ask whether this system is identical with the Lagrange system of an appropriate variational integral. In this article, only a small part of the Euler-Lagrange system may be prescribed in advance. The lack of information does not affect the results. The classical Helmholz solvability conditions and the Tonti resolving formula are adapted for this incomplete problem. Elementary and self-contained algorithmical approach is applied.
\end{abstract}

Keywords: Euler-Lagrange expression; divergence; Helmholz condition; exact inverse problem, differential complex.

MSC 2010: 49N45

\section{Introduction}

In order to introduce our task, let us recall the jet coordinates

$$
x_{i}, w_{I}^{j} \quad\left(i=1, \ldots, n ; j=1, \ldots, m ; I=i_{1} \cdots i_{r} ; r=|I|=0,1, \ldots\right)
$$

named independent variables $x_{1}, \ldots, x_{n}$, dependent variables $w^{1}, \ldots, w^{m}(\operatorname{empty} I=\phi$ with $r=0)$ and higher-order variables $w_{I}^{j}$ (nonempty $I$ ) which correspond to the derivatives

$$
\frac{\partial w^{j}}{\partial x_{I}}=\frac{\partial^{r} w^{j}}{\partial x_{i_{1}} \cdots \partial x_{i_{r}}} \quad\left(I=i_{1} \cdots i_{r} ; i_{1}, \ldots, i_{r}=1, \ldots, n\right)
$$

in the familiar sense. We deal with the local theory of smooth real-valued functions $f=f\left(\cdot \cdot x_{i}, w_{I}^{j}, \cdot \cdot\right)$ depending on a finite number of variables (1) where

$$
\frac{d}{d x_{i}}=\frac{\partial}{\partial x_{i}}+\sum w_{I i}^{j} \frac{\partial}{\partial w_{I}^{j}}, \frac{d}{d x_{I}}=\frac{d}{d x_{i_{1}}} \cdots \frac{d}{d x_{i_{r}}} \quad\left(I=i_{1} \cdots i_{r}\right)
$$

denote the total derivatives.

Let us moreover introduce a novelty, the extended jet space, where the primary coordinates (1) are completed with additional variables

$$
t\left(=x_{n+1}\right), w_{I t}^{j}, w_{I t t}^{j}, \ldots \quad(j, I \text { as above }) .
$$

They are named the parameter variable $t$ and the variations $w_{I t}^{j}, w_{I t t}^{j}, \ldots$ corresponding to the derivatives

$$
\frac{\partial}{\partial t} \frac{\partial w^{j}}{\partial x_{I}}, \frac{\partial^{2}}{\partial t^{2}} \frac{\partial w^{j}}{\partial x_{I}}, \cdots
$$

respectively. If $F=F\left(\cdot, x_{i}, t, w_{I}^{j}, w_{I t}^{j}, \cdot \cdot\right)$ is a function in the extended jet space, the variation operator

$$
\frac{d}{d t}=\frac{\partial}{\partial t}+\sum w_{I t}^{j} \frac{\partial}{\partial w_{I}^{j}}+\sum w_{I t t}^{j} \frac{\partial}{\partial w_{I t}^{j}}+\cdots
$$


can be applied. The variations and total derivatives may be composed and mutually commute. (Later on, the following second extension of the jet space and additional variation denoted either $t=x_{n+2}$ or $s=x_{n+2}$ marginally appears.)

With this preparation, let $f$ and $g$ be functions of variables (1). If we substitute some functions $w^{j}=w^{j}\left(x_{1}, \ldots, x_{n}, t\right)$, then the rule

$$
g w_{I i t}^{j}=-\frac{d g}{d x_{i}} w_{I t}^{j}+\frac{d}{d x_{i}}\left(g w_{I t}^{j}\right)
$$

can be repeatedly applied to provide the variational identity

$$
\frac{d f}{d t}=\sum \frac{\partial f}{\partial w_{I}^{j}} w_{I t}^{j}=\cdots=e[f]+\mathcal{D} \quad\left(e[f]=\sum e^{j}[f] w_{t}^{j}\right)
$$

where

$$
e^{j}[f]=\sum(-1)^{r} \frac{d}{d x_{I}} \frac{\partial f}{\partial w_{I}^{j}} \quad(j=1, \ldots, m), \quad \mathcal{D}=\sum \frac{d}{d x_{i}} F_{i}
$$

are the Euler-Lagrange expressions and the divergence summand (with certain ambigous functions $F_{i}$ ), respectively.

At this place, we recall the actual exact inverse problem of the calculus of variations which is as follows. Certain functions $e^{j}(j=1, \ldots, m)$ of variables $(1)$ are given and we have to decide if there exists a Lagrange function $f$ of variables (1) such that $e^{j}=e^{j}[f]$ for all $j=1, \ldots, m$. This problem is resolved for a long time. We shall however deal with the incomplete version of this problem where not all functions $e^{1}, \ldots, e^{m}$ are prescribed in advance. In accordance with a brief remark in [1], this is a reasonable task which can be effectively investigated if the intermediate terms $\cdots$ in the variational identity (4) are taken into account.

The following remarks should be useful for a better clarity of the article to follow. There are two aspects of the problem, namely the algorithmical and the geometrical one.

We prefer the algorithmical approach which is as follows. There are variables (1) and (2) where the functions of variables (1) can be regarded as functions of variables (2) as well. The procedure (3) applied to a function $f$ of variables (1) provides the identity (4) with certain unique functions $e^{j}[f](j=1, \ldots, m)$ of variables (2). The common inverse problem consists in determination of this $f$ if all functions $e^{j}[f]$ are given. In our article, only a certain part of these functions $e^{j}[f]$ is prescribed. This incomplete problem is in fact very wide, since some properties of the unknown function $f$ can be still postulated in our algorithm.

We intentionally omit the geometrical aspect of the inverse problem. The term "jet space" and "extended jet space" occurring in the article are merely shorter formal substitute for the phrases "the space of variables (1)" and "the space of variables (2)", respectively. We have a good reason for this point of view. Though our article was inspired by geometry [2], the proofs and the final results do not admit any clear geometrical interpretation and cannot be expressed within the framework of the actual jet theories [3],[4]. Roughly, the actual jet theories are formally not appropriate.

In short, the main achievements of the article are as follows. Theorems 2.3 and 2.4 represent the generalized Helmholz conditions and the Tonti resolving formula is improved in Theorem 2.5. After some technical remarks, we discuss the mechanisms of the incomplete inverse problem in the particular case (30)-(36) with only one given function $e^{1}[f]$. This is also illustrated by a few explicit examples. A complete thorough theory would rest on Theorem 2.6 and it does not cause any additional difficulties. We conclude with curious differential complex (57) which latently involves all results of this article.

\section{The Main Achievements}

We start with two well-known and simple results [1].

Proposition 2.1 (uniqueness). Let $f$ be function of variables (1) and

$$
\frac{d f}{d t}=\sum e^{j} w_{t}^{j}+\mathcal{D} \quad\left(\mathcal{D}=\sum \frac{d}{d x_{i}} G_{i}\right)
$$


where $e^{j}$ are functions of variables (1) while $G_{i}$ may depend on variables $(2)$. Then $e^{j}=e^{j}[f]$ for all $j=1, \ldots, m$.

Proposition 2.2 (divergence). The vanishing $e^{j}[f]=0(j=1, \ldots, m)$ is equivalent to the identity

$$
f=\sum \frac{d}{d x_{i}} f_{i}
$$

where $f_{i}$ are appropriate functions of variables (1).

Equations (4) and (5) imply the identity

$$
\sum\left(e^{j}-e^{j}[f]\right) w_{t}^{j}=\sum \frac{d}{d x_{i}}\left(F_{i}-G_{i}\right)
$$

whence the Proposition 2.1 follows by substitution of arbitrary functions $w^{j}=w^{j}\left(x_{1}, \ldots, x_{n}, t\right)$ and subsequent integration over a domain $\Omega$ in the space of variables $x_{1}, \ldots, x_{n}$. Concerning Proposition 2.2, let us insert functions

$$
t w^{j}+(1-t) c^{j} \quad\left(j=1, \ldots, m ; \text { fixed functions } c^{j}=c^{j}\left(\cdot \cdot x_{i}, \cdot \cdot\right)\right)
$$

for all variables $w^{j}$ (and also for $w_{I}^{j}$ and $w_{I t}^{j}$ ) into (4), respectively. Then the integration of border terms provides the identity

$$
f-\left.f\right|_{w^{j}=c^{j}}=\int_{0}^{1} \frac{d f}{d t} d t=\sum \int_{0}^{1} e^{j}[f] d t\left(w^{j}-c^{j}\right)+\sum \frac{d}{d x_{i}} \int_{0}^{1} F_{i} d t .
$$

Assuming moreover $e[f]=0$, we have just the formula (6) where

$$
\left.f\right|_{w^{j}=c^{j}}+\sum \frac{d}{d x_{i}} \int_{0}^{1} F_{i} d t=\sum \frac{d}{d x_{i}} G_{i}=\mathcal{D} \quad\left(\operatorname{certain} G_{i}\right)
$$

clearly is a divergence. Assuming conversely (6), then

$$
\frac{d f}{d t}=\frac{d}{d t} \sum \frac{d}{d x_{i}} f_{i}=\sum \frac{d}{d x_{i}} F_{i} \quad\left(F_{i}=\frac{d}{d t} f_{i}\right)
$$

and the uniqueness implies $e[f]=0$.

Let us turn to the main task. Together with border terms in the variational identity, also the intermediate terms will be taken into account. We focus on one of such intermediate terms $\cdots$ in (4):

$$
\frac{d f}{d t}=\sum \frac{\partial f}{\partial w_{I}^{j}} w_{I t}^{j}=F[f]+\mathcal{D}=e[f]+\mathcal{D} \quad\left(F[f]=\sum e_{I_{j}}^{j}[f] w_{I_{j} t}^{j}\right) .
$$

The sum in the expression $F[f]$ runs over $j=1, \ldots, m$ and all multiindices $I_{j}$ which belong to a certain set $\mathcal{I}(j)$. The vague notation $\mathcal{D}$ for all divergences in the primary jet space is sufficient.

In particular

$$
e_{I_{j}}^{j}[f]=\frac{\partial f}{\partial w_{I_{j}}^{j}}=\frac{\partial f}{\partial w_{I}^{j}}, I_{j} \in \mathcal{I}(j)=\{I:|I| \leq \operatorname{order} f\}, \mathcal{D}=0
$$

for the initial term in (9) and

$$
e_{I_{j}}^{j}[f]=e^{j}[f], I_{j}=\phi, \mathcal{D}=\sum \frac{d}{d x_{i}} F_{i}
$$

for the last term. There are many dissimilar variational identities (9) which correspond to various strategies of the use of the rule (3), we deal with only one such strategy here. 
Theorem 2.3. For any intermediate term, the identity

$$
e[F[f]]=0 \quad\left(F[f]=\sum e_{I_{j}}^{j}[f] w_{I_{j} t}^{j}=\sum e_{I_{j}}^{j}[f] w_{I_{j}, n+1}^{j}\right)
$$

in the extended jet space holds true.

Proof. If $t=x_{n+1}$ is regarded as a mere additional independent variable, Proposition 2.2 can be applied to the equation

$$
\frac{d f}{d t}=\frac{d f}{d x_{n+1}}=F[f]+\mathcal{D}
$$

It follows that

$$
F[f]=\frac{d f}{d x_{n+1}}-\mathcal{D}
$$

is a divergence in the extended jet space which implies $e[F[f]]=0$.

Theorem 2.4. Let certain functions

$$
e_{I_{j}}^{j} \quad\left(j=1, \ldots, m ; I_{j} \in \mathcal{I}(j)\right)
$$

of variables (1) satisfy the identity

$$
e[F]=0 \quad\left(F=\sum e_{I_{j}}^{j} w_{I_{j} t}^{j}=\sum e_{I_{j}}^{j} w_{I_{j}, n+1}^{j}\right)
$$

in the extended jet space. Then

$$
\frac{d f}{d t}=F+\mathcal{D} \quad \text { hence } \quad F=F[f]+\mathcal{D}
$$

for appropriate Lagrange function $f$ of variables (1) and divergence $\mathcal{D}$.

Before passing to the proof, let us mention condition (13) in more detail. In the extended jet space

$$
e[F]=\sum e^{j}[F] w_{t}^{j}=\sum e^{j}[F] w_{n+2}^{j} \quad\left(t=x_{n+2}\right)
$$

with the Euler-Lagrange expressions

$$
e^{j}[F]=\sum(-1)^{r} \frac{d}{d x_{K}} \frac{\partial F}{\partial w_{K}^{j}} \quad\left(j=1, \ldots, m ; K=k_{1} \cdots k_{r} ; r=0,1, \ldots\right)
$$

where $k_{1}, \ldots, k_{r}=1, \ldots, m+1$. Clearly

$$
e^{j^{\prime}}[F]=\sum F_{I}^{j j^{\prime}} w_{I, n+1}^{j} \quad\left(j, I \text { as above } ; j^{\prime}=1, \ldots, m\right)
$$

where the coefficients $F_{I}^{j j^{\prime}}$ are expressed only in terms of functions $e_{I_{j}}^{j}$. It follows that identity (13) is equivalent to the (generalized) Helmholz condition

$$
F_{I}^{j j^{\prime}}=0 \quad\left(\text { all } j \text { and } I \text { as above } ; j^{\prime}=1, \ldots, m\right)
$$

for the given functions $e_{I_{j}}^{j}$.

Proof. In the extended jet space $x_{n+1}$ is taken for the additional independent variable (instead of $t$ ) while $t$ will denote the new variation. So, assuming (13) in the more precise notation, the variational identity for the function $F$ reads

$$
\frac{d F}{d t}=\sum \frac{\partial}{\partial w_{I^{\prime}}^{j^{\prime}}}\left(\sum e_{I_{j}}^{j} w_{I_{j}, n+1}^{j}\right) w_{I^{\prime} t}^{j^{\prime}}+\sum e_{I_{j}}^{j} w_{I_{j}, n+1, t}^{j}=\cdots=\mathbb{D}
$$


since $e[F]=0$. Here

$$
\mathbb{D}=\sum \frac{d}{d x_{i}} G_{i}+\frac{d}{d x_{n+1}} G \quad(\text { sum over } i=1, \ldots, n)
$$

denotes a divergence in the extended jet space. Analogously as in (8), it follows that

$$
F-\left.F\right|_{w^{j}=c^{j}}=\int_{0}^{1} \frac{d F}{d t} d t=\sum \frac{d}{d x_{i}} \int_{0}^{1} G_{i} d t+\sum \frac{d}{d x_{n+1}} \int_{0}^{1} G d t
$$

where (7) was inserted for variables $w^{j}$ before the integration. Altogether

$$
\frac{d f}{d x_{n+1}}=F-\left\{\left.F\right|_{w^{j}=c^{j}}+\sum \frac{d}{d x_{i}} \int_{0}^{1} G_{i} d t\right\} \quad\left(f=\int_{0}^{1} G d t\right) .
$$

Returning to the original notation in the jet space (1), we have the identity

$$
\frac{d f}{d t}=F+\mathcal{D} \quad\left(f=\int_{0}^{1} G d t, \mathcal{D}=-\{\cdots\}\right)
$$

and the proof is done with only one gap: $G$ is a certain function in the extended jet space and we need to prove that $f$ is in fact a function of variables (1).

Theorem 2.5. The Tonti integral

$$
\tilde{f}=\int_{0}^{1} F d t=\sum \int_{0}^{1} e_{I_{j}}^{j} d t\left(w_{I_{j}}^{j}-\frac{\partial c^{j}}{\partial x_{I_{j}}}\right)
$$

can be taken for the Lagrange function $f$ in previous Theorem.

Proof. We shall explicitly calculate the function $G$ in (20) by using the definition equations (17) and (18). For this aim, the rule (3) is applied to the first summand of the middle term in (17) schematically as follows

$$
g w_{I^{\prime} t}^{j^{\prime}}=g w_{i_{1} \cdots i_{r} t}^{j^{\prime}}=\cdots=(-1)^{r} \frac{d g}{d x_{I^{\prime}}} w_{t}^{j^{\prime}}+\mathcal{D}
$$

for the coefficients

$$
g=\frac{\partial}{\partial w_{I^{\prime}}^{j^{\prime}}}\left(\sum e_{I_{j}}^{j} w_{I_{j}, n+1}^{j}\right) .
$$

The resulting summand with the factor $w_{t}^{j^{\prime}}$ belongs to the total sum $e[F]=0$ and may be neglected. The vague summand $\mathcal{D}$ affects only the functions $G_{i}$ in (18) and may be neglected, too. The second summand of the middle term in (17) eventually provides the desired result. Indeed, applying the rule (3) gives

$$
\begin{gathered}
e_{I_{j}}^{j} w_{I_{j}, n+1, t}^{j}=-\frac{d}{d x_{n+1}} e_{I_{j}}^{j} \cdot w_{I_{j} t}^{j}+\frac{d}{d x_{n+1}}\left(e_{I_{j}}^{j} w_{I_{j} t}^{j}\right)=\cdots \\
=(-1)^{\left|I_{j}\right|+1} \frac{d}{d x_{I_{j}}}\left(\frac{d}{d x_{n+1}} e_{I_{j}}^{j}\right) \cdot w_{t}^{j}+\mathcal{D}+(-1)^{\left|I_{j}\right|} \frac{d}{d x_{n+1}}\left(\frac{d}{d x_{I_{j}}} e_{I_{j}}^{j} \cdot w_{t}^{j}+\mathcal{D}\right) .
\end{gathered}
$$

The summand of the final result with the factor $w_{t}^{j}$ again belongs to $e[F]=0$ and may be neglected. The remaining summand provides the solution

$$
G=\sum(-1)^{\left|I_{j}\right|} \frac{d}{d x_{I_{j}}} e_{I_{j}}^{j} \cdot w_{t}^{j}, \quad \tilde{f}=\int_{0}^{1} G d t
$$

which is other than (21). However, instead of this $G$, we can also use the function

$$
\sum(-1)^{\left|I_{j}\right|}(-1)^{\left|I_{j}\right|} e_{I_{j}}^{j} \cdot \frac{d}{d x_{I_{j}}} w_{t}^{j}=\sum e_{I_{j}}^{j} w_{I_{j} t}^{j}=F
$$

since it differs within a mere divergence $\mathcal{D}$. 
Due to the uncertain divergence $\mathcal{D}$ and various possible strategies of calculations, the coefficients $e_{I_{j}}^{j}[f]$ in (9) are not uniquely determined except for the following self-evident case.

Theorem 2.6. For any fixed $j=1, \ldots, m$ with $\mathcal{I}(j)=\{\phi\}$, the coefficients $e_{\phi}^{j}[f]=e^{j}[f]$ of the intermediate term of the variational identity are the Euler-Lagrange expressions.

The connection of Theorems with the incomplete inverse problem will be soon investigated. In the meantime, let us mention some useful technique of calculations to follow later on. Together with the original jet space, we recall the extended jet spaces with independent variables denoted for this time as

$$
x_{1}, \ldots, x_{n}, t\left(=x_{n+1}\right) \quad \text { or } \quad x_{1}, \ldots, x_{n}, t\left(=x_{n+1}\right), s\left(=x_{n+2}\right) .
$$

The parameter variable in the largest second extension is temporarily denoted $s$ instead of $t$ for better clarity. For convenience, we also recall some of the above formulae in the new notation:

$$
\begin{gathered}
F=\sum e_{I_{j}}^{j} w_{I_{j}, n+1}^{j}=\sum e_{I_{j}}^{j} w_{I_{j} t}^{j} \quad\left(j=1, \ldots, m ; I_{j} \in \mathcal{I}(j)\right), \\
e^{j^{\prime}}[F]=\sum(-1)^{|K|} \frac{d}{d x_{K}} \frac{\partial F}{\partial w_{K}^{j^{\prime}}}=\sum F_{I}^{j j^{\prime}} w_{I, n+1}^{j}=\sum F_{I}^{j j^{\prime}} w_{I t}^{j}, \\
e[F]=\sum e^{j^{\prime}}[F] w_{n+2}^{j^{\prime}}=\sum e^{j^{\prime}}[F] w_{s}^{j^{\prime}}=\sum F_{I}^{j j^{\prime}} w_{I t}^{j} w_{s}^{j^{\prime}}
\end{gathered}
$$

where the multiindices $I$ and $I_{j}$ consist of entries $1, \ldots, n$ while $K$ concerns the extendend jet space and entries $1, \ldots, n+1$.

Lemma 2.1. The selfadjointness identity

$$
e[F]=\frac{d F}{d s}-\frac{d G}{d t}+\mathcal{D} \quad\left(F=\sum e_{I_{j}}^{j} w_{I_{j} t}^{j}, G=\sum e_{I_{j}}^{j} w_{I_{j} s}^{j}\right)
$$

holds true with a certain divergence $\mathcal{D}$.

Proof. One can easily find the identity

$$
\sum F_{I}^{j j^{\prime}} w_{I t}^{j}=\sum(-1)^{\left|I^{\prime}\right|} \frac{d}{d x_{I^{\prime}}}\left(\frac{\partial}{\partial w_{I^{\prime}}^{j^{\prime}}} e_{I_{j}}^{j} \cdot w_{I_{j} t}^{j}\right)-\frac{d}{d t} \sum(-1)^{\left|I_{j}\right|} \frac{d}{d x_{I_{j}}} e_{I_{j}}^{j}
$$

by using (23). Moreover clearly

$$
\begin{aligned}
\frac{d}{d s} F & =\sum \frac{\partial}{\partial w_{I^{\prime}}^{j^{\prime}}} e_{I_{j}}^{j} \cdot w_{I^{\prime} s}^{j^{\prime}} w_{I_{j} t}^{j}+\sum e_{I_{j}}^{j} w_{I_{j} t s}^{j}, \\
-\frac{d}{d t} G & =-\sum \frac{\partial}{\partial w_{I^{\prime}}^{j^{\prime}}} e_{I_{j}}^{j} \cdot w_{I^{\prime} t}^{j^{\prime}} w_{I_{j} s}^{j}-\sum e_{I_{j}}^{j} w_{I_{j} s t}^{j} .
\end{aligned}
$$

The multiindices $I^{\prime}$ in the factor $w_{I^{\prime} s}^{j^{\prime}}$ of (27) can be deleted by the rule (3) in order to obtain the factor $w_{s}^{j^{\prime}}$. Analogously, the multiindices $I^{\prime}$ in the factor $w_{I^{\prime} t}^{j^{\prime}}$ can be deleted as well. Then (25) immediately follows with the divergence caused by the already mentioned use of the rule (3).

Lemma 2.2. Helmholz condition (13) is equivalent to the selfadjointness congruence

$$
\frac{d F}{d s} \cong \frac{d G}{d t} \quad(\bmod \mathcal{D}) \quad\left(F=\sum e_{I_{j}}^{j} w_{I_{j} t}^{j}, G=\sum e_{I_{j}}^{j} w_{I_{j} s}^{j}\right) .
$$

This obvious consequences of the previous Lemma 2.2 was known only for the particular "border case" where $F=\sum e^{j} w_{t}^{j}$ and $G=\sum e^{j} w_{s}^{j}$. Then the necessity of (29) becomes easy: the condition $e[F]=0$ implies that

whence

$$
e^{j}=e^{j}[f], F=\frac{d f}{d t}+\mathcal{D}
$$

$$
\frac{d F}{d s}=\frac{d}{d s}\left(\frac{d f}{d t}+\mathcal{D}\right)=\frac{d}{d t}\left(\frac{d f}{d s}+\mathcal{D}\right)=\frac{d G}{d t}+\mathcal{D} .
$$

The sufficience and the general case (29) are much deeper facts. 
Lemma 2.3 (hypothesis). Conditions $F_{I}^{j j^{\prime}}=0$ with $j \leq j^{\prime}$ (or with $j^{\prime} \leq j$ ) imply all the remaining conditions $F_{I}^{j j^{\prime}}=0$ with arbitrary $j$ and $j^{\prime}$.

We can state only a very delicate scheme of the proof. In all applications to follow later on, the Lemma 2.3 will be directly verified if necessary.

First of all, one can find an identity

$$
\left.\sum F_{I}^{j j^{\prime}} w_{I t}^{j} w_{s}^{j^{\prime}}=\sum G_{I}^{j^{\prime} j} w_{I s}^{j^{\prime}} w_{t}^{j}+\mathcal{D} \quad \text { (appropriate } G_{I}^{j^{\prime} j}\right)
$$

by using the rule (3). The divergence $\mathcal{D}$ is a linear combination of various expressions $d F_{I}^{j j^{\prime}} / d x_{I}$, hence the identities $F_{I}^{j j^{\prime}}=0$ imply $G_{I}^{j^{\prime} j}=0$. However the latter identities are also the Helmholz condition with a mere exchanged role of the variations $t$ and $s$. But $j$ is related to $t$ while $j^{\prime}$ is related to $s$. So we may conclude that the Helmholz condition with $j \leq j^{\prime}$ is equivalent to the same conditions with $j^{\prime} \leq j$ hence is equivalent to all Helmholz conditions.

\section{Towards the Inverse Problems}

\section{The initial term}

Let us start with the initial term (10) of the variational identity. Then

$$
e\left[\sum \frac{\partial f}{\partial w_{I}^{j}} w_{I t}^{j}\right]=e\left[\frac{d f}{d t}\right]=0
$$

is trivially satisfied. Conversely assume

$$
e[F]=0 \quad\left(F=\sum e_{I}^{j} w_{I t}^{j}\right)
$$

where $e_{I}^{j}(j=1, \ldots, m ;|I| \leq$ const. $)$ are functions of variables (1). Then

$$
F=\frac{d \tilde{f}}{d t}+\mathcal{D} \quad\left(\tilde{f}=\int_{0}^{1} F d t=\sum \int_{0}^{1} e_{I}^{j} d t\left(w_{I}^{j}-\frac{\partial c^{j}}{\partial x_{I}}\right)\right)
$$

where $(7)$ is inserted into the integral and $c^{j}=c^{j}\left(x_{1}, \ldots, x_{n}\right)$ are arbitrary fixed functions. It should be however noted that condition (B) is always satisfied if the order of variables (1) occuring in functions $e_{I}^{j}$ does not exceed the total length of all multiindices $I$ appearing in the summation of $F$. This easily follows by direct verification. It should be also noted that the existence of function $f$ satisfying the "exact" equations

$$
e_{I}^{j}=\frac{\partial f}{\partial w_{I}^{j}}, F=\sum \frac{\partial f}{\partial w_{I}^{j}} w_{I t}^{j}=\frac{d f}{d t}
$$

is not ensured here.

\section{The last term}

Let us turn to the analogous identity

$$
e\left[\sum e^{j}[f] w_{t}^{j}\right]=e[e[f]]=0
$$

valid for the last term (11). Conversely assume

$$
e[F]=0 \quad\left(F=\sum e^{j} w_{t}^{j}\right)
$$


where $e^{j}(j=1, \ldots, m)$ are certain given functions of variables $(1)$. Then

$$
F=e[\tilde{f}]+\mathcal{D} \quad\left(\tilde{f}=\int_{0}^{1} F d t=\sum \int_{0}^{1} e^{j} d t\left(w^{j}-c^{j}\right)\right) .
$$

However more is true. We have

$$
\frac{d \tilde{f}}{d t}=e[\tilde{f}]+\mathcal{D} \quad \text { whence also } \quad \frac{d \tilde{f}}{d t}=(F-\mathcal{D})+\mathcal{D} \quad(\text { various } \mathcal{D})
$$

by using $(\mathrm{C})$ and the uniqueness applied in the extended jet space implies $e[\tilde{f}]=F+\mathcal{D}$. So we have the familiar solution of the common exact inverse problem: functions $e^{j}(j=1, \ldots, m)$ of variables (1) satisfy the condition (B) if and only if

$$
F=e[f] \text { hence } e^{j}=e^{j}[f](j=1, \ldots, m)
$$

for appropriate $f$ (we may put $f=\tilde{f}$, the Tonti solution).

It is worth mentioning that in our approach, the condition (B) becomes very transparent. Indeed, $e[F]=0$ is satisfied if and only if

$$
e^{j^{\prime}}[F]=e^{j^{\prime}}\left[\sum e^{j} w_{t}^{j}\right]=e^{j^{\prime}}\left[\sum e^{j} w_{n+1}^{j}\right]=0 \quad\left(j^{\prime}=1, \ldots, m\right)
$$

in the extended jet space. In more detail this condition reads

$$
\begin{gathered}
\sum \frac{\partial e^{j}}{\partial w_{0}^{j^{\prime}}} w_{t}^{j}-\sum \frac{d}{d x_{i}}\left(\frac{\partial e^{j}}{\partial w_{i}^{j^{\prime}}} w_{t}^{j}\right)-\frac{d}{d t} e^{j^{\prime}}+ \\
+\sum \frac{d}{d x_{i i^{\prime}}}\left(\frac{\partial e^{j}}{\partial w_{i i^{\prime}}^{j^{\prime}}} w_{t}^{j}\right)-\sum \frac{d}{d x_{i i^{\prime} i^{\prime \prime}}}\left(\frac{\partial e^{j}}{\partial w_{i i^{\prime} i^{\prime \prime}}^{j^{\prime}}} w_{t}^{j}\right)+\cdots=0 .
\end{gathered}
$$

The coefficients $F_{I}^{j j^{\prime}}$ of terms $w_{I t}^{j}$ can be easily found which explicitly provides the Helmholz system (16).

\section{The intermediate terms}

Let us eventually mention the proper intermediate terms of the variational identity. However, there is a legion of various possibilities and a universal theory is hardly realistic at the present time at this place. So we restrict ourselves to a very particular subcase from now on.

We shall be interested only in the identity

$$
e[F[f]]=0 \quad\left(F[f]=e^{1}[f] w_{t}^{1}+\sum_{k \geq 2} \frac{\partial f}{\partial w_{I}^{k}} w_{I t}^{k}\right)
$$

Conversely assuming

$$
e[F]=0 \quad\left(F=e^{1} w_{t}^{1}+\sum_{k \geq 2} e_{I}^{k} w_{I t}^{k}\right)
$$

where $e^{1}, e_{I}^{k}$ are functions of variables (1) then

$$
F=F[\tilde{f}]+\mathcal{D} \quad\left(\tilde{f}=\int_{0}^{1} e^{1} d t\left(w^{1}-c^{1}\right)+\sum \int_{0}^{1} e_{I}^{k} d t\left(w_{I}^{k}-\frac{\partial c^{k}}{\partial x_{I}}\right)\right)
$$

Let us moreover choose $e_{I}^{k}=\partial g / \partial w_{I}^{k}$ which provides the incomplete inverse problem to be discussed. 


\section{The inverse problem}

Abbreviating $e=e^{1}$, we put

$$
F=e w_{t}^{1}+\sum_{k \geq 2} \frac{\partial g}{\partial w_{I}^{k}} w_{I t}^{k}
$$

where $e$ and $g$ are functions of variables (1). Then the condition $e[F]=0$ in the extended jet space ensures the existence of a function $f$ of variables (1) such that

$$
\frac{d f}{d t}=F+\mathcal{D} \quad \text { hence } \quad e=e^{1}[f]
$$

by using Theorems 2.4 and 2.6. Due to Theorem 2.5, one can choose $f=\tilde{f}$ where

$$
\tilde{f}=\int_{0}^{1} F d t=\int_{0}^{1} e d t\left(w^{1}-c^{1}\right)+\sum \int_{0}^{1} \frac{\partial g}{\partial w_{I}^{k}} d t\left(w_{I}^{k}-\frac{\partial c^{k}}{\partial x_{I}}\right)
$$

is the Tonti integral (21). This may be regarded as a solution of the incomplete exact inverse problem with the first Euler-Lagrange expression $e=e^{1}[f]$ given in advance (and function $g$ to be still determined).

In more detail, the solvability requirement $e[F]=0$ reads

$$
e^{j^{\prime}}\left[e w_{t}^{1}+\sum_{j \geq 2} \frac{\partial g}{\partial w_{I}^{j}} w_{I t}^{j}\right]=\sum F_{I}^{j j^{\prime}} w_{I t}^{j}=0 \quad\left(j^{\prime}=1, \ldots, m\right)
$$

where the coefficients $F_{I}^{j j^{\prime}}$ are expressed in terms of functions $e$ and $g$. This provides the generalized Helmholz solvability condition $F_{I}^{j j^{\prime}}=0$ (all $j, j^{\prime}, I$ ) for the functions $e$ and $g$. In our case (30), the coefficients $F_{I}^{j j^{\prime}}$ can be determined by the identity

$$
\begin{aligned}
\sum F_{I}^{j j^{\prime}} w_{I t}^{j} & =\sum(-1)^{\left|I^{\prime}\right|} \frac{d}{d x_{I^{\prime}}}\left(\frac{\partial e}{\partial w_{I^{\prime}}^{j^{\prime}}} \cdot w_{t}^{1}\right)-\frac{d e}{d t}+ \\
+ & \sum(-1)^{\left|I^{\prime}\right|} \frac{d}{d x_{I^{\prime}}}\left(\frac{\partial}{\partial w_{I^{\prime}}^{j^{\prime}}} \frac{\partial g}{\partial w_{I}^{k}} \cdot w_{I t}^{k}\right)-\frac{d}{d t} \sum(-1)^{\left|I^{\prime}\right|} \frac{d}{d x_{I^{\prime}}} \frac{\partial g}{\partial w_{I^{\prime}}^{k}}
\end{aligned}
$$

which is much simpler than the general formula (26).

In the common exact inverse problem where all Euler-Lagrange expressions are given, the Tonti solution is unique. Our incomplete problem is more interesting since the ambigous function $g$ should be still determined. This provides a wide amount of variants of inventions since some properties of $g$ can be apriori postulated. (One can observe that one can even postulate $g=f$ where $f$ is a solution of the inverse problem but it is not the best choice.)

In practice, many technical improvements can be employed. For instance, obviously

$$
F=e w_{t}^{1}-\sum \frac{\partial g}{\partial w_{I}^{1}} w_{I t}^{1}+\frac{d g}{d t}
$$

and it follows that the simplified condition

$$
e^{j^{\prime}}\left[e w_{t}^{1}-\sum \frac{\partial g}{\partial w_{I}^{1}} w_{I t}^{1}\right]=\sum F_{I}^{j j^{\prime}} w_{I t}^{j}=0 \quad\left(j^{\prime}=1, \ldots, m\right)
$$

is equivalent to the previous requirement (33). Warning: the Tonti integral is not simplified.

We will conclude with examples of a mere informative nature without any ambitions on thorough general theory. 


\section{One Independent Variable}

Assuming $n=1$, we abbreviate

$$
\begin{gathered}
x=x_{1}, w_{r}^{j}=w_{1 \cdots 1}^{j}(r \text { terms }), g_{r}^{j}=\frac{\partial g}{\partial w_{r}^{j}}, g_{r r^{\prime}}^{j j^{\prime}}=\frac{\partial^{2} g}{\partial w_{r}^{j} \partial w_{r^{\prime}}^{j^{\prime}}}, \ldots, \\
F=e w_{t}^{1}+\sum_{k \geq 2} g_{r}^{k} w_{r t}^{k}=e w_{t}^{1}-\sum g_{r}^{1} w_{r t}^{1}+\frac{d g}{d t}
\end{gathered}
$$

and then the solvability condition (36) reads

$$
e^{j^{\prime}}\left[e w_{t}^{1}-\sum g_{r}^{1} w_{r t}^{1}\right]=\sum F_{r}^{j j^{\prime}} w_{r t}^{j}=0 \quad\left(j^{\prime}=1, \ldots, m\right) .
$$

In order to determine coefficients $F_{r}^{j j^{\prime}}$, we have either the identity

$$
\begin{aligned}
\sum F_{r}^{j 1} w_{r t}^{j} & =e_{0}^{1} w_{t}^{1}-\sum g_{r 0}^{11} w_{r t}^{1}- \\
& -\frac{d}{d x}\left(e_{1}^{1} w_{t}^{1}-\sum g_{r 1}^{11} w_{r t}^{1}\right)-\frac{d}{d t}\left(e-g_{0}^{1}\right)+ \\
& +\frac{d^{2}}{d x^{2}}\left(e_{2}^{1} w_{t}^{1}-\sum g_{r 2}^{11} w_{r t}^{1}\right)+\frac{d^{2}}{d x d t}\left(-g_{1}^{1}\right)+\cdots
\end{aligned}
$$

or the identity

$$
\sum F_{r}^{j k} w_{r t}^{j}=e_{0}^{k} w_{t}^{1}-\sum g_{r 0}^{1 k} w_{r t}^{1}-\frac{d}{d x}\left(e_{1}^{k} w_{t}^{1}-\sum g_{r 1}^{1 k} w_{r t}^{1}\right)+\cdots
$$

where $k=2, \ldots, m$.

In this example, let us deal with functions

$$
e=e\left(x, \cdot \cdot, w_{0}^{j}, w_{1}^{j}, w_{2}^{j}, \cdot \cdot\right), g=g\left(x, \cdot \cdot, w_{0}^{j}, w_{1}^{j}, \cdot \cdot\right)
$$

of order two and one, respectively, from now on. Then the identity (38) easily provides the sought coefficients

$$
\begin{gathered}
F_{0}^{11}=-\frac{d}{d x} e_{1}^{1}+\frac{d^{2}}{d x^{2}} e_{2}^{1}, F_{1}^{11}=-2 e_{1}^{1}+2 \frac{d}{d x} e_{2}^{1}, F_{r}^{11}=0(r \geq 2), \\
F_{0}^{k 1}=-e_{0}^{k}+g_{00}^{1 k}-\frac{d}{d x} g_{10}^{1 k}, F_{1}^{k 1}=-e_{1}^{k}+g_{01}^{1 k}-\frac{d}{d x} g_{11}^{1 k}-g_{10}^{1 k}, F_{2}^{k 1}=-e_{2}^{k}+g_{11}^{1 k}, \\
F_{r}^{k 1}=0(r \geq 3)
\end{gathered}
$$

where $k=2, \ldots, m$. Quite analogous formulae for the coefficients $F_{r}^{j k}(k=2, \ldots, m)$ can be found by the identity (39), however, they do not provide any further Helmholz conditions. This follows from Lemma 2.3 and can be verified by a little clumsy direct verification omitted here.

It follows that altogether the Helmholz condition consists of the system of requirements

$$
e_{1}^{1}=\frac{d}{d x} e_{2}^{1}, e_{2}^{k}-g_{11}^{1 k}=e_{1}^{k}+g_{10}^{1 k}-g_{01}^{1 k}+\frac{d}{d x} g_{11}^{1 k}=e_{0}^{k}-g_{00}^{1 k}+\frac{d}{d x} g_{10}^{1 k}=0
$$

where $k=2, \ldots, m$. This is a promising result which deserves more analysis, however, we conclude with a few remarks.

The first condition (40) is equivalent to the explicit formula

$$
e=a w_{2}^{1}+\int\left(\frac{\partial a}{\partial x}+\sum \frac{\partial a}{\partial w_{0}^{j}} w_{1}^{j}\right) d w_{1}^{1}+b
$$

where

$$
a=a\left(x, \cdot \cdot, w_{0}^{j}, \cdot \cdot, w_{1}^{1}\right), \quad b=b\left(x, \cdot \cdot, w_{0}^{j}, w_{1}^{k}, w_{2}^{k}, \cdot \cdot\right)
$$


are arbitrary functions of the above mentioned variables. In particular, if function $e$ is independent of all variables $w_{r}^{k}(k=2, \ldots, m)$, we may choose $g=0$ and the Tonti solution

$$
\tilde{f}=\int_{0}^{1} F d t=\int_{0}^{1} e d t\left(w_{0}^{1}-c^{1}\right)
$$

is quite simple. In general, the existence of function $g$ satisfying (40) is not automatically ensured. On the other hand, if such a function $g$ exists then all other functions of this property can be easily determined by resolving the homogeneous system

$$
h_{11}^{1 k}=h_{10}^{1 k}-h_{01}^{1 k}=h_{00}^{1 k}-\frac{d}{d x} h_{10}^{1 k}=0 \quad(k=2, \ldots, m) .
$$

Briefly: the first Euler-Lagrange expression $e=e^{1}[f]$ provides rather thorough overview of all solutions of the exact inverse problem.

\section{Several Independent Variables}

The original multiindices notation is preserved but in order to shorten some formulae, we abbreviate

$$
\begin{gathered}
g_{I}^{j}=\frac{\partial g}{\partial w_{I}^{j}}, g_{I I^{\prime}}^{j j^{\prime}}=\frac{\partial^{2} g}{\partial w_{I}^{j} \partial w_{I^{\prime}}^{j^{\prime}}}, \ldots, g_{0}^{j}=\frac{\partial g}{\partial w^{j}}, g_{I 0}^{j j^{\prime}}=\frac{\partial^{2} g}{\partial w_{I}^{j} \partial w^{j^{\prime}}}, \ldots, \\
F=e w_{t}^{1}+\sum g_{I}^{k} w_{I t}^{k}=e w_{t}^{1}-\sum g_{I}^{1} w_{I t}^{1}+\frac{d g}{d t}
\end{gathered}
$$

and then the solvability condition $(36)$ reads

$$
e^{j^{\prime}}\left[e w_{t}^{1}-\sum g_{I}^{1} w_{I t}^{1}\right]=\sum F_{I}^{j j^{\prime}} w_{I t}^{j}=0 \quad\left(j^{\prime}=1, \ldots, m\right)
$$

Let us deal only with functions $e$ and $g$ of the second order. Coefficients $F_{I}^{j j^{\prime}}$ can be determined from identities

$$
\begin{aligned}
\sum F_{I}^{j 1} w_{I t}^{j} & =e_{0}^{1} w_{t}^{1}-\sum g_{I 0}^{11} w_{I t}^{1}-\sum \frac{d}{d x_{i}}\left(e_{i}^{1} w_{t}^{1}-\sum g_{I i}^{11} w_{I t}^{1}\right)- \\
& -\frac{d}{d t}\left(e-g_{0}^{1}\right)+\sum \frac{d^{2}}{d x_{i} d x_{i^{\prime}}}\left(e_{i i^{\prime}}^{1} w_{t}^{1}-\sum g_{I i i^{\prime}}^{11} w_{I t}^{1}\right)- \\
& +\sum \frac{d^{2}}{d x_{i} d t}\left(-g_{i}^{1}\right)+\sum \frac{d^{3}}{d x_{i} d x_{i^{\prime}} d t} g_{i i^{\prime}}^{1} \\
\sum F_{I}^{j k} w_{I t}^{j} & =e_{0}^{k} w_{t}^{1}-\sum g_{I 0}^{1 k} w_{I t}^{1}-\sum \frac{d}{d x_{i}}\left(e_{i}^{k} w_{t}^{1}-\sum g_{I i}^{1 k} w_{I t}^{1}\right)+ \\
& +\sum \frac{d^{2}}{d x_{i} d x_{i^{\prime}}}\left(e_{i i^{\prime}}^{k} w_{t}^{1}-\sum g_{I i i^{\prime}}^{1 k} w_{I t}^{1}\right)
\end{aligned}
$$

where $k=2, \ldots, m$. As above, identity (44) is enough to provide the Helmholz conditions

$$
\begin{aligned}
& 2 e_{i}^{1}=\sum \frac{d}{d x_{i^{\prime}}} e_{i i^{\prime}}^{1}+\frac{d}{d x_{i}} e_{i i}^{1} \\
& e_{0}^{k}=g_{00}^{1 k}-\sum \frac{d}{d x_{i}} g_{i 0}^{1 k}+\sum \frac{d^{2}}{d x_{i} d x_{i^{\prime}}} g_{i i^{\prime} 0}^{1 k} ; \\
& e_{i}^{k}=g_{0 i}^{1 k}-g_{i 0}^{1 k}+\sum \frac{d}{d x_{i^{\prime}}}\left(g_{i i^{\prime} 0}^{1 k}-g_{i^{\prime} i}^{1 k}\right)+\sum \frac{d^{2}}{d x_{i^{\prime}} d x_{i^{\prime \prime}}} g_{i^{\prime} i^{\prime \prime} i}^{1}{ }^{k} ; \\
& e_{i i}^{k}=g_{0 i i}^{1 k}+g_{i i 0}^{1 k}-g_{i i}^{1 k}+\sum \frac{d}{d x_{i^{\prime}}}\left(g_{i^{\prime} i i}^{1 k}-g_{i i^{\prime} i}^{1 k}\right)+\sum \frac{d^{2}}{d x_{i^{\prime}} d x_{i^{\prime \prime}}} g_{i^{\prime} i^{\prime \prime} i i}^{1}{ }^{k} ; \\
& e_{i i^{\prime}}^{k}=g_{0 i i^{\prime}}^{1 k}+g_{i i^{\prime} 0}^{1 k}-g_{i i^{\prime}}^{1 k}-g_{i^{\prime} i}^{1 k}+\sum \frac{d}{d x_{i^{\prime \prime}}}\left(g_{i^{\prime} i^{\prime \prime} i}^{1, k}-g_{i^{\prime \prime} i i^{\prime}}^{1 k}\right)+ \\
& +\sum \frac{d^{2}}{d x_{i^{\prime \prime}} d x_{i^{\prime \prime \prime}}} g_{i^{\prime \prime} i^{\prime \prime \prime} i i^{\prime}}^{1}
\end{aligned}
$$


where $k=2, \ldots, m$ and $i \neq i^{\prime}$ in the last equation. This is rather instructive result: for given function $e$ satisfying (46), very strong conditions (47) imposed on the function $g$ can be in principle completely analyzed by the common compatibility algorithms. Alas, the calculations are lengthy.

However assume $n, m=2$ and let the function $g$ be of the order one from now on. Then the Helmholz conditions again simplify as

$$
\begin{aligned}
2 e_{1}^{1}=2 \frac{d}{d x_{1}} & e_{11}^{1}+\frac{d}{d x_{2}} e_{12}^{1}, 2 e_{2}^{1}=\frac{d}{d x_{1}} e_{12}^{1}+2 \frac{d}{d x_{2}} e_{22}^{1}, \\
e_{0}^{2} & =g_{00}^{12}-\frac{d}{d x_{1}} g_{10}^{12}-\frac{d}{d x_{2}} g_{20}^{12}, \\
e_{i}^{2} & =g_{i 0}^{21}-g_{0 i}^{21}-\frac{d}{d x_{1}} g_{i 1}^{21}-\frac{d}{d x_{2}} g_{i 2}^{21}, \\
e_{i i}^{2} & =-g_{i i}^{21}, e_{12}^{2}=-g_{21}^{21}-g_{12}^{21}
\end{aligned}
$$

and now we can eventually mention three quite dissimilar examples. The alternative notation $x=x_{1}, y=x_{2}$ will be employed.

First, the function $e=w_{2}^{2} w_{12}^{2}$ satisfies (48). Let us deal only with functions $g=g\left(\cdot \cdot, w_{i}^{j}, \cdot \cdot\right)$. Then the conditions (49) read

$$
\frac{d}{d x} g_{11}^{21}+\frac{d}{d y} g_{12}^{21}=w_{12}^{2}+\frac{d}{d x} g_{21}^{21}+\frac{d}{d y} g_{22}^{21}=g_{11}^{12}=w_{2}^{2}+g_{12}^{12}+g_{21}^{12}=g_{22}^{12}=0 .
$$

The general formula

$$
g=A\left(w_{1}^{1}, w_{2}^{1}\right)+B\left(w_{1}^{2}, w_{2}^{2}\right)+C \cdot\left(w_{1}^{2} w_{2}^{1}-w_{1}^{1} w_{2}^{2}\right)-\frac{1}{2}\left(w_{2}^{2}\right)^{2} w_{1}^{1}
$$

where $A, B$ are arbitrary functions and $C \in \mathbb{R}$ is a constant easily follows. For the particular choice $A=B=C=0$, we obtain the Tonti integral

$$
\begin{aligned}
\tilde{f} & =\int_{0}^{1} e d t\left(w^{1}-c^{1}\right)+\int_{0}^{1} \frac{\partial g}{\partial w_{2}^{2}} d t\left(w_{2}^{2}-\frac{\partial c^{2}}{\partial y}\right)= \\
& =\frac{1}{3} w_{2}^{2} w_{12}^{2}\left(w^{1}-c^{1}\right)-\frac{1}{3} w_{2}^{2} w_{1}^{1}\left(w_{2}^{2}-\frac{\partial c^{2}}{\partial y}\right)
\end{aligned}
$$

where $c^{1}=c^{1}(x, y), c^{2}=c^{2}(x, y)$ may be arbitrary functions. For the choice $c^{1}=c^{2}=0$, clearly

$$
\tilde{f}=\frac{1}{3} w_{2}^{2} w_{12}^{2} w^{1}-\frac{1}{3}\left(w_{2}^{2}\right)^{2} w_{1}^{1}
$$

is a very strange solution. However

$$
f=\tilde{f}-\frac{1}{6} \frac{d}{d x}\left(\left(w_{2}^{2}\right)^{2} w^{1}\right)=-\frac{1}{2}\left(w_{2}^{2}\right)^{2} w_{1}^{1}
$$

again is a solution satisfying $e^{1}[f]=e=w_{2}^{2} w_{12}^{2}$. Briefly: many solutions of the incomplete exact inverse problem can be explicitly calculated and the Tonti integral need not be the "most economical" one.

Second, the function $e=w_{11}^{1} w_{22}^{1}-\left(w_{12}^{1}\right)^{2}$ satisfies (48). Let us again deal with functions $g=g\left(\cdot \cdot, w_{i}^{j}, \cdot \cdot\right)$. Conditions (49) are simple and need not be stated here. They are satisfied if

$$
g=A\left(w_{1}^{1}, w_{2}^{2}\right)+B\left(w_{1}^{2}, w_{2}^{2}\right)+C \cdot\left(w_{1}^{2} w_{2}^{1}-w_{1}^{1} w_{2}^{2}\right)
$$

where $A, B$ and $C \in \mathbb{R}$ are arbitrary. For the particular case $A=B=C=0$ we obtain the Tonti solution $\tilde{f}=e w^{1}$. 
Third, let us conclude with the function $e=0$ identically vanishing and all possible functions $g=g\left(x, y, \cdot \cdot, w^{j}, w_{i}^{j}, \cdot \cdot\right)$ of the first order. Conditions (49) read

$$
\begin{gathered}
g_{00}^{21}=\frac{d}{d x} g_{01}^{21}+\frac{d}{d y} g_{02}^{21}, \\
g_{10}^{21}-g_{01}^{21}=\frac{d}{d x} g_{11}^{21}+\frac{d}{d y} g_{12}^{21}, g_{20}^{21}-g_{02}^{21}=\frac{d}{d x} g_{21}^{21}+\frac{d}{d y} g_{22}^{21}, \\
g_{11}^{21}=g_{21}^{21}+g_{12}^{21}=g_{22}^{21}=0 .
\end{gathered}
$$

Requirements (52) seem to be easy. Indeed, they are satisfied if

$$
g=A\left(w_{1}^{1}, w_{2}^{1}\right)+B\left(w_{1}^{2}, w_{2}^{2}\right)+C\left(w_{1}^{1}, w_{2}^{2}\right)+D\left(w_{1}^{2}, w_{2}^{1}\right),
$$

where

$$
\begin{aligned}
& C=E w_{2}^{2} w_{1}^{1}+\bar{C} w_{2}^{2}+\tilde{C} w_{1}^{1}+c, \\
& D=-E w_{1}^{2} w_{2}^{1}+\bar{D} w_{2}^{1}+\tilde{D} w_{1}^{2}+d .
\end{aligned}
$$

Functions $A, B$ and coefficients $E, \ldots, d$ in (53) depend also on variables $x, y, w^{1}, w^{2}$ which is not explicitly declared here. Then the requirements (51) simplify as

$$
\tilde{D}_{0}^{1}-\tilde{C}_{0}^{2}+E_{y}=A_{10}^{12}-B_{10}^{21}, \bar{C}_{0}^{1}-\bar{D}_{0}^{2}-E_{x}=A_{20}^{12}-B_{20}^{21}
$$

and imply the formulae

$$
A=\bar{A} w_{1}^{1}+\tilde{A} w_{2}^{1}+a, B=\bar{B} w_{1}^{2}+\tilde{B} w_{2}^{2}+b
$$

with the identities

$$
\tilde{D}_{0}^{1}-\tilde{C}_{0}^{2}+E_{y}=\bar{A}_{0}^{2}-\bar{B}_{0}^{1}, \bar{C}_{0}^{1}-\bar{D}_{0}^{2}-E_{x}=\tilde{A}_{0}^{2}-\tilde{B}_{0}^{1} .
$$

All coefficients $\bar{A}, \ldots, b$ in (54) again depend on variables $x, y, w^{1}, w^{2}$. The remaining requirement (50) eventually provides the final identities

$$
E_{y}=\tilde{C}_{0}^{2}-\tilde{D}_{0}^{1}, E_{x}=\bar{D}_{0}^{2}-\bar{C}_{0}^{1},\left((c+d)_{0}^{1}-\tilde{C}_{x}-\tilde{D}_{y}\right)_{0}^{2}=0 .
$$

Altogether taken,

$$
\begin{aligned}
g & =\bar{A} w_{1}^{1}+\tilde{A} w_{2}^{1}+a+\bar{B} w_{1}^{2}+\tilde{B} w_{2}^{2}+b+E \cdot\left(w_{2}^{2} w_{1}^{1}-w_{1}^{2} w_{2}^{1}\right)+ \\
& +\bar{C} w_{2}^{2}+\tilde{C} w_{1}^{1}+c+\bar{D} w_{2}^{1}+\tilde{D} w_{1}^{2}+d
\end{aligned}
$$

by using (53) and (54). It follows that we may suppose $\bar{A}=\tilde{A}=a=\bar{B}=\tilde{B}=b=d=0$ without any loss of generality. Then the final identities reduce to the underdetermined system of equations

$$
\tilde{C}_{0}^{2}-\tilde{D}_{0}^{1}=E_{y}, \bar{C}_{0}^{1}-\bar{D}_{0}^{2}=E_{x},\left(c_{0}^{1}-c_{x}\right)_{0}^{2}=0
$$

for the functions $\tilde{C}, \bar{C}, \tilde{D}, \bar{D}, E, c$ of variables $x, y, w^{1}, w^{2}$. It can be easily resolved by explicit formulae.

\section{A Complement}

For every $n=0,1, \ldots$ and a fixed $m=1,2, \ldots$, let $\mathbf{F}_{n}$ denote the module of all smooth functions of a finite number of variables (1). Since $\mathbf{F}_{0} \subset \mathbf{F}_{1} \subset \cdots$, the mapping

$$
e[f]=\sum e^{j}[f] w_{n+1}^{j} \in \mathbf{F}_{n+1} \quad\left(f \in \mathbf{F}_{n}\right)
$$

is not rigorously defined. But let us introduce a label $n$ : if $\mathbf{C}_{n}$ denotes the module of all couples $\{f, n\}$ where $f \in \mathbf{F}_{n}$, then the mappings

$$
d_{n}: \mathbf{C}_{n} \rightarrow \mathbf{C}_{n+1}, d_{n}\{f, n\}=\left\{\sum e^{j}[f] w_{n+1}^{j}, n+1\right\}
$$

make a good sense. We obtain even a differential complex

$$
\mathbf{C}_{0} \stackrel{d_{0}}{\longrightarrow} \mathbf{C}_{1} \longrightarrow \cdots \longrightarrow \mathbf{C}_{n} \stackrel{d_{n}}{\longrightarrow} \mathbf{C}_{n+1} \longrightarrow \cdots \quad\left(d_{n+1} d_{n}=0\right)
$$


equivalent to the classical Helmholz solvability condition

$$
e[e[f]]=e\left[\sum e^{j}[f] w_{n+1}^{j}\right]=0 \quad\left(f \in \mathbf{F}_{n}\right) .
$$

(The initial differential $d_{0}$ is not involved but this is an easy matter since

$$
f=f\left(\cdot \cdot w^{j}, \cdot \cdot\right), e[f]=\sum \frac{\partial f}{\partial w^{j}} w_{1}^{j}=\frac{d f}{d x_{1}}, e\left[\frac{d f}{d x_{1}}\right]=0,
$$

if $f \in \mathbf{F}_{0}$.) All the above results of this article can be interpreted by certain properties of the complex (57).

For instance, let us recall the function

$$
F=\sum e_{I_{j}}^{j} w_{I_{j}, n+1}^{j} \in \mathbf{F}_{n+1} \quad\left(e_{I_{j}}^{j} \in \mathbf{F}_{n}, I_{j} \in \mathcal{I}(j)\right)
$$

of Theorem 2.4. Then $d_{n+1}\{F, n+1\}=0$ if and only if there exists a function $f \in \mathbf{F}_{n}$ such that $F=F[f]+\mathcal{D}$ hence

$$
F-F[f] \in \mathbf{F}_{n}, d_{n}\{F-F[f], n\}=0 .
$$

Here $F[f]$ can be any intermediate term of the variational identity $(9)$, however, if the set $\mathcal{I}(j)=\phi$ is empty for certain $j$ then the unique term $e_{I_{j}}^{j}=e_{\phi}^{j}=e^{j}$ is just the Euler-Lagrange expression. This is the idea of our solution of the incomplete inverse problem.

The complex (57) is of a very strange nature. It concerns all Lagrange functions with various number of independent variables. The common differential complexes occuring in the mathematical analysis and differential topology are of quite other kind.

Acknowledgments. This paper was elaborated with the financial support of the European Union's "Operational Programme Research and Development for Innovations", No. CZ.1.05/2.1.00/03.0097, as an activity of the regional Centre AdMaS "Advanced Materials, Structures and Technologies".

\section{References}

1. V. Chrastinová and V. Tryhuk. On the exact inverse problem of the calculus of variations. Advances in Analysis, 2(3):196-218, 2017.

2. Jan Chrastina. Inverse problem of the classical calculus of variations. Arch. Math. (Brno), 18(1):9-14, 1982.

3. D. Krupka and D. Saunders, editors. Handbook of global analysis. Elsevier Science B.V., Amsterdam, 2008.

4. D. Krupka. The Sonin-Douglas problem. In The inverse problem of the calculus of variations, Atlantis Stud. Var. Geom., chapter 2, pages 31-73. Atlantis Press, Paris, 2015. 